\title{
Sedation in non-invasive ventilation: do we know what to do (and why)?
}

\author{
Dan Longrois ${ }^{1 *}$, Giorgio Conti ${ }^{2}$, Jean Mantz ${ }^{3}$, Andreas Faltlhauser ${ }^{4}$, Riku Aantaa ${ }^{5}$ and Peter Tonner ${ }^{6}$
}

\begin{abstract}
This review examines some of the issues encountered in the use of sedation in patients receiving respiratory support from non-invasive ventilation (NIV). This is an area of critical and intensive care medicine where there are limited (if any) robust data to guide the development of best practice and where local custom appears to exert a strong influence on patterns of care.

We examine aspects of sedation for NIV where the current lack of structure may be contributing to missed opportunities to improve standards of care and examine the existing sedative armamentarium. No single sedative agent is currently available that fulfils the criteria for an ideal agent but we offer some observations on the relative merits of different agents as they relate to considerations such as effects on respiratory drive and timing, and airways patency. The significance of agitation and delirium and the affective aspect(s) of dyspnoea are also considered.

We outline an agenda for placing the use of sedation in NIV on a more systematic footing, including clearly expressed criteria and conditions for terminating NIV and structural and organizational conditions for prospective multicentre trials.
\end{abstract}

Keywords: Agitation, Benzodiazepines, Delirium, Dexmedetomidine, Dyspnoea, Ketamine, Non-invasive ventilation, Opioids, Propofol, Sedation

\section{Introduction}

Use of non-invasive ventilation (NIV) in critical and intensive care has expanded greatly in recent decades in response to evidence of its benefits as a means of reducing dependence on invasive (i.e. with tracheal intubation) ventilation and associated complications, and for the management of acute respiratory failure. Adoption and application of NIV are nevertheless still in a phase of evolution [1]. We examine this subject with specific reference to the use and impact of sedation during NIV.

This essay examines a non-exhaustive series of questions about NIV and the possible role of sedation as a co-intervention to NIV. The answers - to the extent that answers are available - emerge from sources that range from evidence-based medicine to expert opinion. This range is itself indicative of the incomplete nature of our current understanding of these matters.

\footnotetext{
* Correspondence: dan.longrois@bch.aphp.fr

'Université Paris-Diderot, Hôpitaux Universitaires Paris Nord Val de Seine, Département d'Anesthésie Réanimation Chirurgicale, Hôpital Bichat-Claude Bernard, Paris, France

Full list of author information is available at the end of the article
}

\section{Review}

Is sedation universal or mandatory during NIV?

A non-exhaustive inspection of recent reports invites the conclusion that we as physicians are either indifferent to or unconcerned about the general topic of sedation during NIV. The authors of the most recent Cochrane review on the place of NIV as a weaning strategy for intubated adults with respiratory failure reported that only one of the studies they identified used a standardized sedation protocol before or after initiation of NIV (Table 1) and argued that the role of sedation as a co-intervention requires specific investigation in future trials [2-8]. Separately, Scala has identified only eight small clinical studies of sedation in NIV, in which a total of 183 patients were assigned to seven different sedative drug regimens, the effects of which were assessed by multiple different and non-congruent outcome parameters (Tables 2 and 3) [9-17].

The drift of these observations appears to be that either sedation has not been perceived as a major problem or opportunity within the wider context of NIV use or that it has not been studied systematically. The first of 
Table 1 Summary of studies making any report of sedation use in the context of NIV in adults with critical illness (compiled from Burns et al. [2])

\begin{tabular}{|c|c|c|c|c|}
\hline Study & $\begin{array}{c}\text { No. of } \\
\text { patients }\end{array}$ & Patient characteristics & Experimental NIV strategy & Sedation status \\
\hline $\begin{array}{l}\text { Hill et al. } \\
2000[4]\end{array}$ & 21 & ARF & VPAP in ST-A mode & $\begin{array}{l}\text { Sedation protocol reportedly used. Study published } \\
\text { as abstract only }\end{array}$ \\
\hline $\begin{array}{l}\text { Nava et al. } \\
1998[5]\end{array}$ & 50 & $\begin{array}{l}\text { Exacerbation of COPD; } \\
\text { mechanical ventilation } \\
\text { for at least } 36-48 \mathrm{~h}\end{array}$ & $\begin{array}{l}\text { Non-invasive pressure support } \\
\text { on conventional ventilator } \\
\text { delivered with face mask }\end{array}$ & $\begin{array}{l}\text { Sedation reportedly used during NIV but apparently } \\
\text { not protocolized or defined }\end{array}$ \\
\hline $\begin{array}{l}\text { Prasad et al. } \\
2009[6]\end{array}$ & 30 & COPD; AHRF & $\begin{array}{l}\text { Bilevel NIV (pressure mode) } \\
\text { delivered by full face mask }\end{array}$ & $\begin{array}{l}\text { Patients received neuromuscular blocking drugs and } \\
\text { sedatives in immediately preceding phase of invasive } \\
\text { ventilation. Use of sedation during NIV not clear }\end{array}$ \\
\hline $\begin{array}{l}\text { Rabie Agmy } \\
\text { et al. } 2004 \text { [7] }\end{array}$ & 37 & Exacerbation of COPD & $\begin{array}{l}\text { Proportional-assist NIV in } \\
\text { timed mode, delivered by } \\
\text { face or nasal mask }\end{array}$ & $\begin{array}{l}\text { Patients received neuromuscular blocking drugs and } \\
\text { sedatives in immediately preceding phase of invasive } \\
\text { ventilation. Use of sedation during NIV not clear }\end{array}$ \\
\hline $\begin{array}{l}\text { Vaschetto et al. } \\
2012 \text { [8] }\end{array}$ & 20 & $\begin{array}{l}\text { Hypoxaemic respiratory } \\
\text { failure; invasive mechanical } \\
\text { ventilation for at least } 48 \mathrm{~h}\end{array}$ & Helmet NIV & $\begin{array}{l}\text { Sedation reportedly used during NIV but apparently not } \\
\text { protocolized or defined. Rates of continuous sedation during NIV } \\
\text { reported to be similar in both groups (a priori study outcome) }\end{array}$ \\
\hline
\end{tabular}

In all the studies, the control strategy used was invasive pressure support.

ARF, acute respiratory failure; AHRF, acute hypercapnic respiratory failure; ST, spontaneous/timed; VPAP, variable positive airway pressure.

these positions may have led to the second; alternatively, the lack of studies may reflect complaisance (if not complacency) about this aspect of NIV technique and procedure. In any event, both these positions suggest a lack of attention to the possibilities in this area of NIV.

Narrowly considered, therefore, the answer to our own question must be "We do not know". However, the findings of the only survey of practice that we have identified make it clear that in reality the answer is "No", as only $\approx$ $25 \%$ of NIV patients received sedation (rising to $\approx 40 \%$ in critical care) [18]. Whether or not that percentage represents an appropriate level of sedation use among recipients of NIV is just one of many questions to which there is at present no definitive answer. Inspection of Table 2 suggests that, in those studies where sedation during NIV was explicitly addressed, the main reason for sedation was "poor NIV acceptance". One general aspect of poor NIV acceptance could be related to the patient-device interface, i.e. the type of mask that is used and also the assisted ventilation pattern. The choice of NIV interface may influence the need for sedation. While it can be argued that "the clinical efficacy of different masks is on average very similar" [19], our experience is aligned with reports that patient acceptance is greatest with the least constricting interfaces such as the helmet - and declines as more intrusive forms of mask are used [20]. As for the breathing modes, bilevel positive airway pressure often produces a need for anxiolysis or sedation whereas spontaneous breathing patterns such as continuous positive airways pressure seldom require such interventions.

In summary, sedation is not mandatory for NIV but it may help in specific situations. There are at present no explicit guiding principles or simple formulae to identify those situations.

Table 2 Studies of sedation in NIV (compiled from Scala [9])

\begin{tabular}{|c|c|c|c|c|c|}
\hline Study & No. of patients & Indication & NIV interface & Type of sedative & Initiation of sedation \\
\hline Rocker et al. [10] & 10 & ARF & FFM & Morphine & At start of NIV \\
\hline Constantin et al. [11] & 13 & $\begin{array}{l}\operatorname{ARF}(n=10) \\
\operatorname{AHRF}(n=3)\end{array}$ & FFM & Remifentanil, midazolam* & Poor NIV acceptance \\
\hline Rocco et al. [12] & 36 & ARF & FFM, helmet & Remifentanil & Poor NIV acceptance \\
\hline Akada et al. [13] & 10 & ARF & FFM & Dexmedetomidine $^{\dagger}$ & Poor NIV acceptance \\
\hline Takasaki et al. [14] & 2 & SAA & FFM & Dexmedetomidine & Poor NIV acceptance \\
\hline Clouzeau et al. [15] & 10 & $\begin{array}{l}\operatorname{ARF}(n=7) \\
\operatorname{AHRF}(n=3)\end{array}$ & FFM & Propofol & Poor NIV acceptance \\
\hline Senoglu et al. [16] & 40 & COPD & FFM & Dexmedetomidine $(n=20)$; midazolam $(n=20)$ & At start of NIV \\
\hline Huang et al. [17] & 62 & ACPO & FFM, helmet & Dexmedetomidine $(n=33) ;$ midazolam $(n=29)$ & Poor NIV acceptance \\
\hline
\end{tabular}

The last two rows identify randomized controlled trials; other trials were reported not to have been controlled. See also Table 3 of this review. ACPO, acute cardiogenic pulmonary oedema; ARF, acute respiratory failure; AHRF, acute hypercapnic respiratory failure; FFM, full face mask; SAA, severe asthma attack.

${ }^{*}$ Combined with propofol in three cases. ${ }^{\dagger}$ Combined with morphine in one case and with propofol in one case. 
Table 3 Reported dosages of sedatives administered in studies of sedation in NIV (compiled from Scala [9])

\begin{tabular}{llll}
\hline Drug & No. of patients & Dosage & Sedation target range \\
\hline Dexmedetomidine & 41 & $1 \mu \mathrm{g} / \mathrm{kg}$ (bolus); 0.2-0.7 $\mu \mathrm{g} / \mathrm{kg} / \mathrm{h}$ (infusion) & RSS 2-3; RASS 2-4; BIS >85 \\
Midazolam & 41 & $0.05 \mathrm{mg} / \mathrm{kg}$ (bolus); 0.05-0.1 mg/kg/h (infusion) & RSS 2-3; RASS 2-4; BIS >85 \\
Remifentanil & 38 & $0.025-0.1 \mu \mathrm{g} / \mathrm{kg} / \mathrm{min}$ (infusion) & RSS 2-3 \\
Propofol & 43 & $0.4 \mu \mathrm{g} / \mathrm{mL}$ (target serum concentration; step-down to $0.2 \mu \mathrm{g} / \mathrm{mL}$ ) & OAAS $/ \mathrm{S} 3-4$ \\
\hline
\end{tabular}

The information in this Table is derived from references 10-17. See also Table 2 of this review.

BIS, bispectral index; OAAS/S, Observer's Assessment of Alertness/Sedation Scale, RASS, Richmond Agitation and Sedation Scale; RSS, Ramsey Sedation Scale.

\section{Does NIV affect sedation goals?}

The goals of sedation for a cooperative patient in the intensive care unit (ICU) are to provide analgesia and comfort, preserve day/night cycles (including natural sleep) and avoid nuisances such as ambient light and noise. Additional goals include haemodynamic stability, preservation of metabolic homeostasis, muscular relaxation, preservation of diaphragmatic function and attenuation of the stress/immune response, as well as considerations such as programmed withdrawal from sedation: they should be no different during NIV.

Nevertheless, if we regard NIV as a stage in the progression from intermittent mandatory ventilation to spontaneous breathing then there must be a parallel expectation of a progressive reduction in use of sedation. Two other points need to be discussed for sedation during NIV. Firstly, the avoidance of the respiratory depressant effects of different sedatives; secondly, untoward effects of sedative drugs on the upper airway, a topic that has received new impetus with increased awareness of obstructive sleep apnoea [21-24].

Therefore the answer to this question is "Yes", both quantitatively (i.e. less 'deep' sedation) and qualitatively in that sedation during NIV must be performed with no/ minimal respiratory depression and no/minimal impairment of the upper airway.

\section{Is sedation per se a factor contributing to the success or failure of NIV?}

Patient acceptance and compliance are essential to the success of NIV [13]. Achieving patient acceptance and compliance is a multifaceted exercise, with staff proficiency and competence being one major influence [25]. However, acceptance/compliance also relate directly to sedation since neither can be expected from an insensate patient; nor are they likely to be forthcoming if the patient is anxious, agitated or disoriented. The necessity of a sedation regimen that brings the patient to a state of calm, alert cooperation is clearly implied by these considerations.

Any decision to resort to sedation must be taken as the last stage in a careful evaluation of the causes of actual or pending failure, as outlined in Figure 1. It must also take account of the fact that the likely success of NIV in hypoxaemic respiratory failure varies considerably according to the presenting condition [26] and that there is no robust evidence that sedation will materially affect situations where the response rate to NIV is intrinsically poor. Indeed, adding sedation in these situations may be disadvantageous by obscuring a failure of NIV due to underlying pathology and thus delaying a necessary intubation. Similarly, sedation does not obviate any of the contraindications to NIV [26].

Nava and Ceriana [25] have partitioned NIV failure into immediate ( $<1 \mathrm{~h}$ after commencement), early (1-48 h) and late $(>48 \mathrm{~h})$ and identified predictors of failure for each time segment. Factors implicated in immediate NIV

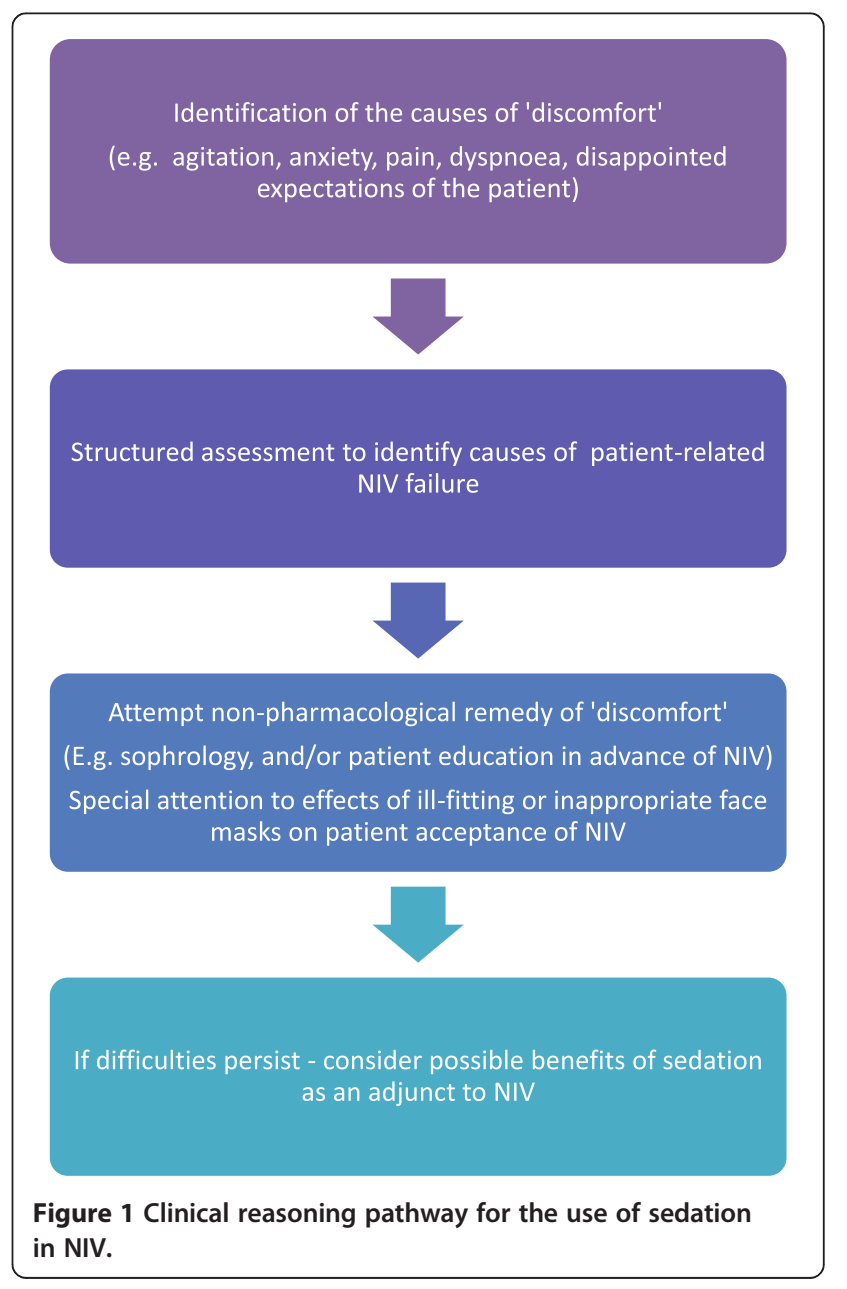


failure include "intolerance, agitation, and patient-ventilator asynchrony", for which "judicious sedation" is recommended but not characterized in detail.

Sleep disruption was identified as a factor in late NIV failure [25]. The subject of sleep and sleep disruption in ICU patients has attracted much research in recent years (see for example Cabello et al. [27] and Roche Campo et al. [28]) and one instinctively inclines to the belief that more normal patterns and quality of sleep are part of the restoration of health. Whether this is indeed the case is harder to demonstrate than might be expected but investigations of the differential effects of sedatives on electrophysiological dimensions of sleep provide food for thought [29]. This is, of course, an area where environmental alterations (i.e. minimizing noise and disturbance) may also have an important influence and such measures should always precede any use of sedatives.

In mechanically ventilated patients, ventilator asynchrony may adversely affect sleep [30]: the extent to which this disruptive interaction may work in the opposite direction is not clear, but there is evidence that, in NIV patients, sleep is associated with more asynchrony and blood-oxygen desaturations than the awake state [31]. These data were obtained from a small cohort of patients receiving long-term NIV at home and probably do not fully illuminate the situation of ICU patients receiving both NIV and sedatives. Choice/type of ventilator is also an important influence on asynchrony [32,33] and, at least in some cases, is likely to be more significant than use or non-use of sedatives. Similarly, ventilators with specific leak compensation modes and the ability to generate flows $\geq 10 \mathrm{~L} / \mathrm{min}$ provide better patient compliance than ICU ventilators with little leak adaptive capacity.

The answer to the question is thus that we do not know for certain but that it is unlikely that sedation can 'rescue' poorly configured or inappropriate NIV. However, we hypothesize that adequate sedation can improve patient comfort in specific situations (see below).

\section{What types of patients/specific situations might benefit from sedation during NIV?}

Reference to English language guidelines for NIV [1] reveals that the range of indications for which there is compelling or even strongly persuasive (Grade 2B or better) evidence for benefit of NIV is small and may be summarized as follows.

Acute respiratory failure in the forms of:

- Acute-on-chronic exacerbation of chronic obstructive pulmonary disease (COPD) with acidotic and hypercapnoeic features

- Respiratory failure secondary to cardiogenic pulmonary oedema not arising from shock or acute coronary syndrome
- Acute respiratory failure in immunocompromised patients.

As an adjunct to extubation (but only in expert centres) for:

- Patients who have COPD

- Patients considered to be at high risk of recurrent respiratory failure.

We would expect most candidates for sedation during NIV to come from these categories, and hence to have certain common presenting features.

As noted above, discomfort, anxiety, agitation, pain, dyspnoea and the disappointed expectations of the patient are central in many cases to failure of NIV and hence also to the decision to use sedation in NIV. Delirium may also be a consideration. Use of sedation in NIV is, in this context, firmly within the sphere of the clinical practice guidelines for the management of pain, agitation, and delirium [34] and many of the provisions of those guidelines are relevant, most especially the obligation to identify and correct (by non-pharmacological means if feasible) the causes of pain, agitation, etc.

\section{Agitation and delirium}

A first category to consider is patients who are already agitated before tracheal extubation. A systematic investigation of the causes of anxiety should precede any prescription of a sedative drug. If all otherwise correctable causes of anxiety are eliminated, and anxiety sine materia or anxiety due to a decrease/change in sedative regimen is diagnosed, a case may be made (albeit on the basis of very slender clinical experience) for dexmedetomidine as an aid to extubation [35]. The mechanisms underlying this benefit are not clear and require further, systematic investigation.

The impact of delirium in patients receiving NIV is urgently in need of attention. The most substantial report we have found identified a high prevalence of delirium in NIV patients $(\approx 37 \%)$ and linked that to a marked increase in risk of NIV failure. However, the data on which these findings were based were described as "scarce and of low quality" [36]. The role of sedation in the promotion or prevention of ICU delirium has attracted much comment in recent years but firm conclusions are still hard to come by. For the moment, we are unable to go beyond the pain, agitation, and delirium guidelines, which offer weak (Grade 2B) endorsement for dexmedetomidine in delirium management. "Prevention" of delirium rests substantially on non-pharmacological methods, particularly early mobilization. Where sedation is used at all, an emphasis on early mobilization implies a sedative regimen that facilitates patient participation. (See Nydahl et al. [37] for a recent perspective on this matter). 


\section{Dyspnoea}

A second category of patients could be those who are dyspnoeic and anxious, dyspnoea being associated with delay of extubation [38]. The neuro(patho)physiology and clinical aspects of dyspnoea have been examined in detail in a report of the American Thoracic Society that stratifies dyspnoea according to the quality of the dyspnoea experience, the stimuli that evokes it and the afferent neuronal pathways that mediates it [39].

A full discussion of this matter is beyond the scope of this review but it is worth noting that there is an affective component of dyspnoea that may be differentiated from the sensory dimension and might be amenable to independent manipulation [40-43]. This signals the importance of identifying and appraising the anxiety component of dyspnoea. Self-evidently, the cooperation of the patient is needed for this and any existing sedation regimen must be adapted to that need.

The affective dimension of dyspnoea may be investigated using either single-item ratings of severity of distress or unpleasantness or multi-item scales of emotional responses such as anxiety $[39,44,45]$. However, there are a vast array of dyspnoea rating scales, which address different aspects of the condition. This is not a reason not to measure dyspnoea but it is essential to specify which scale is used for the purpose and to recognize that the nature of any intervention on dyspnoea is likely to be determined (or at least influenced) by the aspects of dyspnoea privileged by the chosen scale.

Hence, sedation could be of benefit in situations where NIV is clearly indicated and where careful evaluation identifies anxiety, dyspnoea with a high affective dimension or delirium as barriers to its successful implementation.

\section{Are there evidence-based reasons to prefer specific sedative drugs during NIV?}

There are no robust data to favour any one drug, class of drugs or protocol over all others. Some of the criteria that may shape the selection of sedatives for NIV are summarized in Table 4. No drug or class fully satisfies all these criteria and the final decision rests for the moment on a sequence of clinical reasoning.

We fully endorse the general presumption against benzodiazepines expressed in the pain, agitation, and delirium guidelines. It is, therefore, a matter of concern that the data of Devlin et al. suggest that benzodiazepines retain a hold in NIV [18]: however, those data are 7 years old and may not represent current habits. Fresh research into prescribing patterns (and the reasoning underpinning them) is desirable: study of the effects of switching sedatives would also be illuminating. Patterns of sedation when NIV is delivered on standard wards are undocumented as far as we know and this is another area that deserves more attention.

Given the pathophysiology of NIV failure, at least three aspects could be influenced by the choice of sedative drugs: the patency of the upper airway, respiratory depression and the affective dimension of dyspnoea. From a pharmacological point of view, dexmedetomidine appears to offer the range of qualities best configured to address these concerns (see for example Hsu et al. [46]). However, the evidence from controlled trials is not sufficient to give this conclusion the force of a guideline and additional considerations (see Table 4) may shape the final selection of drugs for individual patients. (See also Ho et al. [47] for a cautionary case report).

Effects on airways patency and the timing and drive of respiration have been examined earlier in this review and make a case against benzodiazepines (and perhaps other $\gamma$-aminobutyric acid-ergic agents) and possibly in favour of dexmedetomidine. Opioids and benzodiazepines decrease upper airway diameter and are probably deleterious during NIV. Propofol has also been shown to increase the collapsibility of the upper airway in a dose/concentrationdependent manner [23].

Dexmedetomidine has no direct effects on the patency of the upper airways. When used as adjunct therapy it may reduce requirements for opioids (or other sedatives) and so reduce the likelihood of opioid-induced compromise of the upper airways. Among the sedative drugs,

Table 4 Properties of sedative drug classes relevant to delivery of sedation in NIV

\begin{tabular}{|c|c|c|c|c|c|c|c|c|c|c|}
\hline Sedative & $\begin{array}{l}\text { Haemodynamic } \\
\text { stability }\end{array}$ & Analgesia & Amnesia & Anxiolysis & PVD & $\begin{array}{l}\text { Avoidance } \\
\text { of PONV }\end{array}$ & $\begin{array}{l}\text { Promotion of } \\
\text { natural sleep }\end{array}$ & $\begin{array}{l}\text { Suitability for use } \\
\text { after extubation }\end{array}$ & $\begin{array}{l}\text { Delirium } \\
\text { avoidance }\end{array}$ & Total \\
\hline Propofol & 2 & 2 & 2 & 2 & 2 & 4 & 2 & 2 & 1 & 20 \\
\hline Midazolam & 3 & 2 & 4 & 2 & 2 & 2 & 2 & 1 & 1 & 19 \\
\hline Opioids & 4 & 4 & 1 & 2 & 1 & 1 & 1 & 2 & 1 & 20 \\
\hline Dexmedetomidine & 3 & 2 & 2 & 4 & 4 & 2 & 4 & 4 & 3 & 28 \\
\hline Ketamine & 4 & 3 & 2 & 1 & 4 & 1 & 1 & 4 & 1 & 21 \\
\hline
\end{tabular}

Larger numbers indicate a more satisfactory impact on the nominated property. This is primarily a qualitative and relative assessment of the features and benefits of different drugs and drug classes, framed in general terms. Hence, the individual category scores and in particular scores shown in the 'Total' column are crude summaries that should not be over-interpreted and which do not necessarily reflect the net merits or demerits of particular agents in the circumstances of a particular patient.

PONV, postoperative nausea and vomiting; PVD, preservation of ventilatory drive. 
dexmedetomidine has the lowest risk of depression of the respiratory centres.

Ketamine does not cause respiratory depression at doses given for analgesia or procedural sedation [48]. Furthermore, it decreases airway resistance, improves dynamic compliance and preserves functional residual capacity, minute ventilation and tidal volume, while retaining protective pharyngeal and laryngeal reflexes [49]. Ketamine can produce hypersalivation and emergence reactions [49]. Because of its effects on the sympathetic nervous system, ketamine should not be used in decompensated heart failure (typically cardiogenic pulmonary oedema in the context of NIV). There is a relatively abundant literature concerning the use of ketamine for procedural sedation [50], but experience for sedation during NIV is practically non-existent.

The answer to this question, therefore, is that sedative drugs used during NIV should have properties that further the goals of sedation during NIV.

From a pharmacological point of view, benzodiazepines should be avoided: outcome studies in mechanically ventilated patients are consistent with that view but there are no similar trials in NIV to support definitive guidance [51]. (Other recent work provides a timely reminder that without a robust experimental structure it is difficult to make meaningful comparisons between sedatives [52]).

Dexmedetomidine and ketamine seem to have the most suitable overall pharmacological profiles. Propofol and opioids (such as remifentanil) are in an intermediate position.

\section{Conclusions}

Much of the use of sedation in NIV appears to be empirical and is perhaps unstructured. Above all, it appears to be under-researched: we may be doing better than we realize or we may be doing less well than we could. With the continuing growth of NIV as a clinical resource there is a need for sedation practice to be put on a more systematic footing.

To that end we propose a three-point plan.

(1) Repeat the international survey of 2007 [18] to ascertain how, if at all, patterns of sedation use in NIV have changed in the intervening years and, if possible, identify the drivers of change.

(2) Review best practice frameworks in NIV to ensure that guidance includes indications for sedation use, standardized sedation protocols and clearly expressed criteria and conditions for terminating NIV.

(3) Develop at least one (preferably more) prospective multicentre trial on the effects of sedation in NIV. Such a trial needs to enrol substantially more patients than have hitherto been recruited to such studies and needs to incorporate the principles identified in point (2) and a set of unambiguous, informative and reliable endpoints, including a predefined and generally accepted definition of NIV failure. The work of Huang et al. [17] provides a useful, though not exhaustive, template in this respect.

In addition to standardization of sedation protocols, such a trial needs to apply consistent and predefined NIV modalities. Given the need for substantial numbers of patients, we anticipate that such a study would, in the first instance, be confined to patients with acute exacerbation of COPD, as this is the only category likely to yield sufficient patients within an acceptable and practicable period of time.

An alternative or additional possibility would be a prospective trial of NIV to prevent acute respiratory failure in patients recovering from cardiac, abdominal or possibly thoracic surgery [53,54]. Given that these patients would be emerging from surgical anaesthesia, there might be a larger role for sedation and that would facilitate rapid recruitment.

\section{Competing interests}

DL reports honoraria from Orion Pharma for guest lectures at international symposia in anaesthesia and intensive care and editorship of intensetimes, an electronic journal supported by Orion Pharma.

GC reports honoraria for lectures from Orion Pharma (Italy), Covidien and Sylcomed. The Catholic University of Rome has received an institutional research grant from Orion Pharma.

JM reports honoraria from Orion Pharma as a guest lecturer at international symposia in intensive care.

AF reports travel and educational grants from Orion Pharma, CSL Behring and Pulsion Medical Systems and research grants from Pulsion Medical Systems and Köhler Chemie.

RA has been a paid consultant for Orion Corporation (Espoo, Finland) and Abbott Laboratories (Abbott Park, IL, USA), the original co-developers of dexmedetomidine, and also for Hospira (Lake Forest, IL, USA). Hospira has a license agreement with Orion Corporation concerning dexmedetomidine (Precedex). He is also one of the three original patent holders of "Use of dexmedetomidine for sedative effect in patients in an intensive care unit" (WO/1999/049854)

PT reports honoraria for lectures at national and international symposia from Orion Pharma, Abbvie, Baxter, Bard, B. Braun and Ratiopharm.

\section{Authors' contributions}

All authors contributed equally to the discussions that gave rise to this article. All authors contributed equally to the development of the final manuscript and approved the final manuscript.

\section{Acknowledgements}

The authors thank Alexander Scott (Leeds, UK), Dorte Bulow Keld (Aarhus, Denmark), Alberto Sandiumenge (Tarragona, Spain), Ilona Bubek (Budapest, Hungary) and Per-Olof Joachimson (Uppsala, Sweden) for their contributions to the roundtable debate that provided the intellectual framework of this article. The roundtable was made possible through the financial support of Orion Pharma, Espoo, Finland.

Editorial preparation of this manuscript was assisted by Hughes associates, Oxford, UK, on behalf of Orion Pharma.

\section{Author details}

'Université Paris-Diderot, Hôpitaux Universitaires Paris Nord Val de Seine, Département d'Anesthésie Réanimation Chirurgicale, Hôpital Bichat-Claude 
Bernard, Paris, France. '2Department of Intensive Care and Anesthesiology, Università Cattolica del Sacro Cuore, Rome, Italy. ${ }^{3}$ Anesthesiology Department, Beaujon Hospital, AP-HP, Université Paris-Diderot, Paris, France. ${ }^{4}$ First Department of Internal Medicine, Kliniken Nordoberpfalz AG, Klinikum Weiden, Weiden, Germany. ${ }^{5}$ Department of Anesthesiology, Intensive Care, Emergency Care and Pain Medicine, University of Turku, Turku, Finland. ${ }^{6}$ Department of Anesthesiology and Intensive Care Medicine, Emergency Medicine Hospital Links der Weser GmbH, Bremen, Germany.

Received: 25 September 2014 Accepted: 6 October 2014

Published: 4 November 2014

\section{References}

1. Keenan SP, Sinuff T, Burns KE, Muscedere J, Kutsogiannis J, Mehta S, Cook DJ, Ayas N, Adhikari NK, Hand L, Scales DC, Pagnotta R, Lazosky L, Rocker G, Dial S, Laupland K, Sanders K, Dodek P, Canadian Critical Care Trials Group/ Canadian Critical Care Society Noninvasive Ventilation Guidelines Group: Clinical practice guidelines for the use of noninvasive positive-pressure ventilation and noninvasive continuous positive airway pressure in the acute care setting. CMAJ 2011, 183:E195-E214.

2. Burns KE, Meade MO, Premji A, Adhikari NK: Noninvasive ventilation as a weaning strategy for mechanical ventilation in adults with respiratory failure: a Cochrane systematic review. CMAJ 2014, 186:E112-E122.

3. Burns KE, Meade MO, Premji A, Adhikari NK: Noninvasive positive-pressure ventilation as a weaning strategy for intubated adults with respiratory failure. Cochrane Database Syst Rev 2013, 12, CD004127.

4. Hill NS, Lin D, Levy M, O'Brien A, Klinger J, Houtchens J, Nelson D, McCormick J: Noninvasive positive pressure ventilation (NPPV) to facilitate extubation after acute respiratory failure: a feasibility study [abstract]. Am J Respir Crit Care Med 2000, 161:B18.

5. Nava S, Ambrosino N, Clini E, Prato M, Orlando G, Vitacca M, Brigada P, Fracchia C, Rubini F: Noninvasive mechanical ventilation in the weaning of patients with respiratory failure due to chronic obstructive pulmonary disease: a randomized, controlled trial. Ann Intern Med 1998, 128:721-728.

6. Prasad SB, Chaudhry D, Khanna R: Role of noninvasive ventilation in weaning from mechanical ventilation in patients of chronic obstructive pulmonary disease: an Indian experience. Indian J Crit Care Med 2009, 13:207-212.

7. Rabie Agmy GM, Mohamed AZ, Mohamed RN: Noninvasive ventilation in the weaning of patients with acute-on-chronic respiratory failure due to COPD. Chest 2004, 126(Suppl 4):755.

8. Vaschetto R, Turucz E, Dellapiazza F, Guido S, Colombo D, Cammarota G, Della Corte F, Antonelli M, Navalesi P: Noninvasive ventilation after early extubation in patients recovering from hypoxemic acute respiratory failure: a single-centre feasibility study. Intensive Care Med 2012, 38:1599-1606

9. Scala R: Sedation during non-invasive ventilation to treat acute respiratory failure. Shortness of Breath 2013, 2:35-43.

10. Rocker GM, Mackenzie MG, Williams B, Logan PM: Noninvasive positive pressure ventilation: successful outcome in patients with acute lung injury/ARDS. Chest 1999, 115:173-177.

11. Constantin JM, Schneider E, Cayot-Constantin S, Guerin R, Bannier F, Futier E, Bazin JE: Remifentanil-based sedation to treat noninvasive ventilation failure: a preliminary study. Intensive Care Med 2007, 33:82-87.

12. Rocco M, Conti G, Alessandri E, Morelli A, Spadetta G, Laderchi A, Di Santo C, Francavilla S, Pietropaoli P: Rescue treatment for non invasive ventilation failure due to interface intolerance with remifentanilanalgo sedation: a pilot study. Intensive Care Med 2010, 36:2060-2065.

13. Akada S, Takeda S, Yoshida Y, Nakazato K, Mori M, Hongo T, Tanaka K, Sakamoto A: The efficacy of dexmedetomidine in patients with noninvasive ventilation: a preliminary study. Anesth Analg 2008, 107:167-170.

14. Takasaki Y, Kido T, Samba K: Dexmedetomidine facilitates induction of noninvasive positive pressure ventilation for acute respiratory failure in patients with severe asthma. J Anesth 2009, 23:147-150.

15. Clouzeau B, Bui HN, Vargas F, Grenouillet-Delacre M, Guilhon E, Gruson D, Hilbert G: Target-controlled infusion of propofol for sedation in patients with non-invasive ventilation failure due to low tolerance: a preliminary study. Intensive Care Med 2010, 36:1675-1680.

16. Senoglu N, Oksuz H, Dogan Z, Yildiz H, Demirkiran H, Ekerbicer H: Sedation during noninvasive mechanical ventilation with dexmedetomidine or midazolam: A randomized, double-blind, prospective study. Curr Ther Res Clin Exp 2010, 71:141-153.

17. Huang Z, Chen YS, Yang ZL, Liu JY: Dexmedetomidine versus midazolam for the sedation of patients with non-invasive ventilation failure. Intern Med 2012, 51:2299-2305.

18. Devlin JW, Nava S, Fong JJ, Bahhady I, Hill NS: Survey of sedation practices during noninvasive positive-pressure ventilation to treat acute respiratory failure. Crit Care Med 2007, 35:2298-2302.

19. Sferrazza Papa GF, Di Marco F, Akoumianaki E, Brochard L: Recent advances in interfaces for non-invasive ventilation: from bench studies to practical issues. Minerva Anestesiol 2012, 78:1146-1153.

20. Chawla R, Sidhu US, Kumar V, Nagarkar S, Brochard L: Noninvasive ventilation: a survey of practice patterns of its use in India. Indian J Crit Care Med 2008, 12:163-169.

21. Zhuang PJ, Wang X, Zhang XF, Zhou ZJ, Wang Q: Postoperative respiratory and analgesic effects of dexmedetomidine or morphine for adenotonsillectomy in children with obstructive sleep apnoea. Anaesthesia 2011, 66:989-993.

22. Ankichetty S, Wong J, Chung F: A systematic review of the effects of sedatives and anesthetics in patients with obstructive sleep apnea. J Anaesthesiol Clin Pharmacol 2011, 27:447-458.

23. Eastwood PR, Platt PR, Shepherd K, Maddison K, Hillman DR: Collapsibility of the upper airway at different concentrations of propofolanesthesia. Anesthesiology 2005, 103:470-477.

24. Olofsen E, Boom M, Nieuwenhuijs D, Sarton E, Teppema L, Aarts L, Dahan A: Modeling the non-steady state respiratory effects of remifentanil in awake and propofol-sedated healthy volunteers. Anesthesiology 2010, 112:1382-1395.

25. Nava S, Ceriana P: Causes of failure of noninvasive mechanical ventilation. Respir Care 2004, 49:295-303.

26. Glossop AJ, Shephard N, Bryden DC, Mills GH: Non-invasive ventilation for weaning, avoiding reintubation after extubation and in the postoperative period: a meta-analysis. Br J Anaesth 2012, 109:305-314.

27. Cabello B, Thille AW, Drouot X, Galia F, Mancebo J, d'Ortho MP, Brochard L: Sleep quality in mechanically ventilated patients: comparison of three ventilatory modes. Crit Care Med 2008, 36:1749-1755.

28. Roche Campo F, Drouot X, Thille AW, Galia F, Cabello B, d'Ortho MP, Brochard L: Poor sleep quality is associated with late noninvasive ventilation failure in patients with acute hypercapnic respiratory failure. Crit Care Med 2010, 38:477-485.

29. Brown EN, Lydic R, Schiff ND: General anesthesia, sleep, and coma. N Engl J Med 2010, 363:2638-2650

30. Bosma K, Ferreyra G, Ambrogio C, Pasero D, Mirabella L, Braghiroli A, Appendini L, Mascia L, Ranieri VM: Patient-ventilator interaction and sleep in mechanically ventilated patients: pressure support versus proportional assist ventilation. Crit Care Med 2007, 35:1048-1054.

31. Fanfulla F, Taurino AE, Lupo ND, Trentin R, D'Ambrosio C, Nava S: Effect of sleep on patient/ventilator asynchrony in patients undergoing chronic non-invasive mechanical ventilation. Respir Med 2007, 101:1702-1707.

32. Carteaux G, Lyazidi A, Cordoba-Izquierdo A, Vignaux L, Jolliet P, Thille AW, Richard JC, Brochard L: Patient-ventilator asynchrony during noninvasive ventilation: a bench and clinical study. Chest 2012, 142:367-376.

33. Vignaux L, Tassaux D, Carteaux G, Roeseler J, Piquilloud L, Brochard L, Jolliet $P$ : Performance of noninvasive ventilation algorithms on ICU ventilators during pressure support: a clinical study. Intensive Care Med 2010, 36:2053-2059

34. Barr J, Fraser GL, Puntillo K, Ely EW, Gélinas C, Dasta JF, Davidson JE, Devlin JW, Kress JP, Joffe AM, Coursin DB, Herr DL, Tung A, Robinson BR, Fontaine DK, Ramsay MA, Riker RR, Sessler CN, Pun B, Skrobik Y, Jaeschke R, American College of Critical Care Medicine: Clinical practice guidelines for the management of pain, agitation, and delirium in adult patients in the intensive care unit. Crit Care Med 2013, 41:263-306.

35. Arpino PA, Kalafatas $K$, Thompson BT: Feasibility of dexmedetomidine in facilitating extubation in the intensive care unit. J Clin Pharm Ther 2008, 33:25-30.

36. Charlesworth M, Elliott MW, Holmes JD: Noninvasive positive pressure ventilation for acute respiratory failure in delirious patients: understudied, underreported, or underappreciated? A systematic review and meta-analysis. Lung 2012, 190:597-603.

37. Nydahl P, Ruhl AP, Bartoszek G, Dubb R, Filipovic S, Flohr HJ, Kaltwasser A, Mende H, Rothaug O, Schuchhardt D, Schwabbauer N, Needham DM: Early 
mobilization of mechanically ventilated patients: a 1-day point-prevalence study in Germany. Crit Care Med 2014, 42(5):1178-1186.

38. Schmidt M, Demoule A, Polito A, Porchet R, Aboab J, Siami S, Morelot-Panzini C, Similowski T, Sharshar T: Dyspnea in mechanically ventilated critically ill patients. Crit Care Med 2011, 39:2059-2065.

39. Parshall MB, Schwartzstein RM, Adams L, Banzett RB, Manning HL, Bourbeau J, Calverley PM, Gift AG, Harver A, Lareau SC, Mahler DA, Meek PM, O'Donnell DE, American Thoracic Society Committee on Dyspnea: An official American Thoracic Society statement: update on the mechanisms, assessment, and management of dyspnea. Am J Respir Crit Care Med 2012, 185:435-452.

40. Banzett RB, Mulnier HE, Murphy K, Rosen SD, Wise RJ, Adams L: Breathlessness in humans activates insular cortex. Neuroreport 2000, 11:2117-2120

41. Peiffer C, Poline JB, Thivard L, Aubier M, Samson Y: Neural substrates for the perception of acutely induced dyspnea. Am J Respir Crit Care Med 2001, 163:951-957.

42. Evans KC, Banzett RB, Adams L, McKay L, Frackowiak RS, Corfield DR: BOLD fMRI identifies limbic, paralimbic, and cerebellar activation during air hunger. J Neurophysiol 2002, 88:1500-1511.

43. von Leupoldt A, Dahme B: Differentiation between the sensory and affective dimension of dyspnea during resistive load breathing in normal subjects. Chest 2005, 128:3345-3349.

44. von Leupoldt A, Ambruzsova R, Nordmeyer S, Jeske N, Dahme B: Sensory and affective aspects of dyspnea contribute differentially to the Borg scale's measurement of dyspnea. Respiration 2006, 73:762-768.

45. Yorke J, Russell AM, Swigris J, Shuldham C, Haigh C, Rochnia N, Hoyle J, Jones PW: Assessment of dyspnea in asthma: validation of The Dyspnea- 12 . J Asthma 2011, 48:602-608.

46. Hsu Y-W, Cortinez LI, Robertson KM, Keifer JC, Sum-Ping ST, Moretti EW, Young CC, Wright DR, Macleod DB, Somma J: Dexmedetomidine pharmacodynamics: part I. crossover comparison of the respiratory effects of dexmedetomidine and remifentanil in healthy volunteers. Anesthesiology 2004, 101:1066-1076.

47. Ho AM-H, Chen S, Karmakar MK: Central apnoea after balanced general anaesthesia that included dexmedetomidine. Br J Anaesth 2005, 95:773-775.

48. Morel DR, Forster A, Gemperle M: Noninvasive evaluation of breathing pattern and thoraco-abdominal motion following the infusion of ketamine or droperidol in humans. Anesthesiology 1986, 65:392-398,

49. Miller $\mathrm{AC}$, Jamin $\mathrm{CT}$, Elamin EM: Continuous intravenous infusion of ketamine for maintenance sedation. Minerva Anestesiol 2011, 77:812-820.

50. Parashchanka A, Schelfout S, Coppens M: Role of novel drugs in sedation outside the operating room: dexmedetomidine, ketamine and remifentanil. Curr Opin Anaesthesiol 2014, 27:442-447.

51. Jakob SM, Ruokonen E, Grounds RM, Sarapohja T, Garratt C, Pocock SJ, Bratty JR, Takala J, Dexmedetomidine for Long-Term Sedation Investigators: Dexmedetomidine vs midazolam or propofol for sedation during prolonged mechanical ventilation: two randomized controlled trials. JAMA 2012, 307:1151-1160.

52. Devlin JW, Al-Qadheeb NS, Chi A, Roberts R, Qawi I, Garpestad E, Hill NS: Efficacy and safety of early dexmedetomidine during non-invasive ventilation for patients with acute respiratory failure: a randomized, double-blind, placebo-controlled, pilot study. Chest 2014, 145:1204-1212.

53. Chiumello D, Chevallard G, Gregoretti C: Non-invasive ventilation in postoperative patients: a systematic review. Intensive Care Med 2011, 37:918-929.

54. Jaber S, Chanques G, Jung B: Postoperative noninvasive ventilation. Anesthesiology 2010, 112:453-461.

doi:10.1186/2049-6958-9-56

Cite this article as: Longrois et al: Sedation in non-invasive ventilation: do we know what to do (and why)? Multidisciplinary Respiratory Medicine 2014 9:56.

\section{Submit your next manuscript to BioMed Central and take full advantage of:}

- Convenient online submission

- Thorough peer review

- No space constraints or color figure charges

- Immediate publication on acceptance

- Inclusion in PubMed, CAS, Scopus and Google Scholar

- Research which is freely available for redistribution 